\title{
After School Tangram as an Example in Interactive Digital Puzzle Game Design
}

\author{
P. S. $\mathrm{Pa}^{+}$and C. W. Sun \\ Department of Digital Content Design, Graduate School of Toy and Game Design, National Taipei \\ University of Education, Taipei, Taiwan
}

\begin{abstract}
The study is to discuss the effect which is to combine both Entity toys and Digital game. Tangram is one of the common entity toys. We use Tangram and the image recognition to integrate Entity toys and Digital game in order to raise the interest of Puzzle game. Players have to put Tangram together on a platform with a video camera filming. We can use the image recognition to identify if the players have completed the mission. It shows the performance evaluation of the combination of Entity toys and Digital game compared with the traditional entity toys based on the result of the games. Research Performance: 1 . For Human-Computer Interaction, we design a digital game platform. By using a webcam, the computer will be notified the position how players place the pieces of Tangram and identify if the mission is completed. 2 . For the digital game platform, Players not only play Tangram with the traditional game rules, but also have to complete the mission within a limited time. It can increase the interest of Puzzle game by adding the game stages. 3. By doing the surveys, we conclude the primary purpose of the game development and try to understand what Players want and learn from Tangram to raise their interest to the game. After Players finish playing the game, they will do a survey again to show if the game combined both Entity toys and Digital can satisfy their request to raise the interest to Puzzle game indeed. Moreover, the survey result can be an efficient research source for the future Puzzle game design. Human-Computer Interaction game is getting popular recently. The combination of Entity toys and Digital gives a new definition to the ancient puzzle games. Playing Tangram with this new combination can get more people to enjoy the game easily.
\end{abstract}

Keywords: digital game, interaction, puzzle game, Tangram, players, toys.

\section{Introduction}

Digital games are developing rapidly in this age of information. From family consoles and arcade machines to online games, new developments are constantly being introduced. The definition of games is quite broad as well. According to Jesper Juul [1-2], games have six attributes:

1) A formal rule system

2) Variable, quantifiable outcome

3) Different games have different values

4) Player efforts exert an influence on the game

5) Player is emotionally attached to the outcome of the game

6) The consequences of the game are variable and negotiable

Apart from the definition of games, the gaming process is particularly important during game design. Pagulayan et al. [3] mentioned that games should focus on the process of playing the game rather than the outcome. The game should also impose many restrictions that help to increase the interest of the game. The game designed in this study will conform to the six definitions of games proposed by Jesper Juul (2003) and use restrictions to offer players different gaming interests. The following will sort through game types and

\footnotetext{
+ Corresponding author. Tel.: +886-910668143.

E-mail address: myhow@ seed.net.tw
} 
the nature of interaction. Advances in digital technology means interactive gaming has become increasingly important in gaming or related digital products. With more and more interactive products to choose from however the quality of interaction becomes even more important [4-5]. S.Y. Ye (2010) believed that there are three common factors that influence the quality of interaction on whether designing games or software interaction: number of choices, interaction speed, and interaction complexity. Interactive games must not only pay attention to the quality of interaction as mentioned above [6-7]. The human-machine interaction (HMI) of interactive games has drawn increasing attention in recent years as well. HMI enables effective and convenient control by the user. That is, there must be sufficient and appropriate communication between the user and the computer [8-9]. A broader definition of HMI is that all forms of communication between humans and technology can be defined as HMI [10]. Novak (2004) even divided the interaction model in games into four different types [11]:

1) Player to game

2) Player to player

3) Player to developer

4) Player to platform

For interaction design, this study will focus on the response time of the game as a whole while also paying attention to the pacing of the overall game. For the interactive device, video-conferencing will be used to realize player-to-game interaction. A score ranking mechanism will be used to promote interaction between players while hardware will be used to expand platform functionality and player interaction. The overall HMI sensor design will take into account the five following areas (Bellotti et al., 2002) and be realized through complementary software and hardware [12-15]:

1) Address: How to enable communication between one or multiple devices with the system.

2) Attention: How to know that the system is ready.

3) Action: How to control the target and make meaningful operations.

4) Alignment: How to determine the correct response.

5) Accident: How to avoid mistakes.

Traditional puzzle games can be played in different ways but they lack light \& sound effects as well as the fun of multi-player competition. When digital versions of puzzle games began to emerge, using the keyboard and mouse to play puzzle games not only added more interesting audio effects and animation but also boosted the fun of multi-player competition. What it lacks however is the "hands-on" fun of traditional puzzle games because the game is played through just keyboard and mouse. This study will combine digital puzzle gaming with a human-machine interface to design an interactive digital puzzle game. A questionnaire survey analysis will also be used explore the level of player acceptance and the difference with traditional puzzle games. This study hopes to digitize puzzle games as well as identify new concepts and interactive control methods in order to realize an integrated software and hardware design. Tangram is most often associated with mathematics. The different shapes of the tangram can be mathematically computed to determine the alternative for each piece. Mathematics is what most people have the greatest exposure to during their schooling. The focus is therefore on placed on the classroom and the story of the game is an extension of the classroom.

\section{Game Design}

\subsection{System structure}

The overall software and hardware architecture of the After School Tangram is as shown in Figure 1. Control is affected over the USB connecting the software and hardware. The hardware consists of the Phidget control panel, servo motor and webcam all controlled directly via Flash AS3.0. The Phidget Control Panel is used for supplementary testing of Phidget components.

\subsection{Game theme}


Puzzle games generally have different themes to increase buyer and player interest. A version of the well-known puzzle game Klotski for example features characters from the Romance of the Three Kingdoms on the gaming pieces. In recent years, an adaptation of Klotski for the mobile platform saw each piece turned into different cars. During the concept stage, themes that players have most or frequent contact with were chosen as the basic concept. The tangram is most often associated with mathematics and the different shapes of the tangram can be mathematically computed to determine the alternative for each piece. Mathematics is what most people have the greatest exposure to during their schooling. The focus is therefore on placed on the classroom and the story of the game is an extension of the classroom.

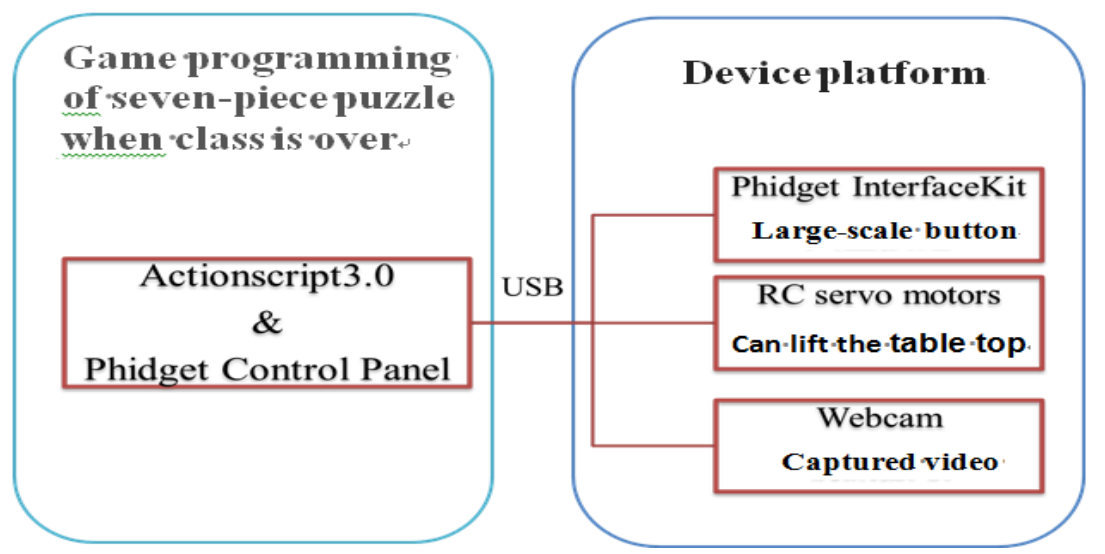

Fig. 1: Overall game software and hardware architecture of After School Tangram.

\section{Game Mechanism}

The game mechanisms designed for this study shall be described below. Different mechanisms will be tailored to different requirements in order to increase the interactive elements in the game. The three main mechanisms are the decision, timing, and question mechanisms. The full program will be included in Appendix 4.

\subsection{Decision Mechanism}

The overall gaming mechanism is based on decisions of the shapes assembled by the player. There are many ways of using the webcam for shape recognition including: gray-scale processing and image value testing. The processing of image values takes up more system resources leading to longer decision times and slowing down the pace of the game. This study therefore uses the capture of special points and the color values at these points for shape recognition. Special point recognition is based on capturing an image pixel value for every decision point for every tangram scenario in order to determine if a tangram exists as shown in Figure 2. In other words, the capture points needed for decision-making are defined in advance.

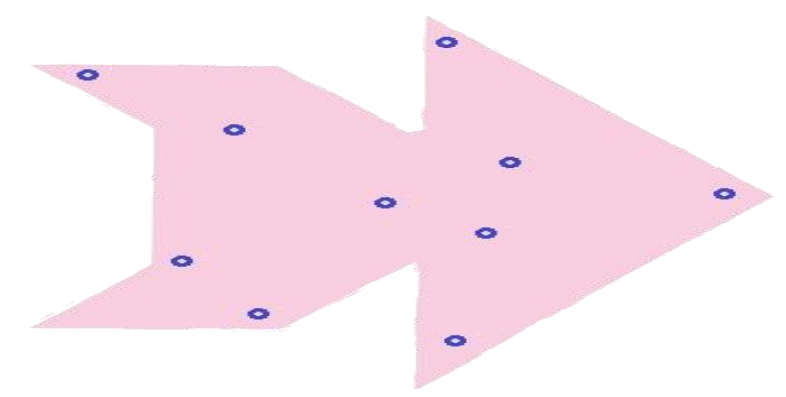

Fig. 2: Illustration of the decision points.

There are 10 decision points for each scenario. Though an image pixel is captured at each point, to avoid judgment errors due to sampling only one pixel at each point the values of the surrounding 8 pixels are sampled as well. A total of 90 pixels are therefore used for decision-making. This was the value decided upon after much testing to ensure performance on even low-end computers without sacrificing game 
performance and pace. The sampled pixel value is used as the cut-off value. In this game, decisions are based on whether the pixel value is closer to black or white. A pixel that is closer to black will have a value that tends towards zero. If it is closer to white than the value will be higher. The setting of the cut-off value must be based on the environmental lighting. During configuration, it is necessary to detect the pixel value of the game platform and the pixel value of the tangram then use the median value for configuration. Each decision point has nine pixel values. If at least six exceeds the cut-off value then there is a tangram piece at this decision point.

\subsection{Timing and question mechanisms}

Time pressure is a mechanism added by this study for the tangram. The player must not only come up with a solution to the tangram scenario but also complete all of the levels within the given time. Time pressure is a common element in many games. From a commercial perspective, including time as an element can be used to control play time and increase the number of times that a game is replayed by players. It also makes the tangram more challenging and sets it apart from conventional tangram games. To avoid players from becoming familiar with scenarios after multiple sessions, scenarios at chosen at random from an existing pool of questions.

\subsection{Interactive mechanisms}

The interactive mechanisms of this study is consists of obstacles and movable platform. The obstacle component interferes with the player's hand movements towards the latter parts of the game while leaving tangram pieces already placed on the platform alone. This increases game difficulty and player interaction. As for the movable platform, when the game enters a new level, the platform tips the tangram pieces by the player's hand to make it easy for them to pick up during the game. The game device consists of the tangram pieces and movable game platform. The design and setup will be described below.

(1) Tangram

The tangram used in this study is cut from wooden boards. The size of each tangram piece is a proportional representation of tangram pieces cut to size. To retain the texture of the wood, the design does not call for the pieces to be painted or treated in any way. As a result, under normal lighting the pixel values of the wooden board tends toward white. This just happens to match the design requirements of the pixel cut-

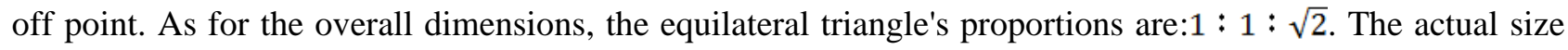
of the tangram pieces was scaled accordingly. This made it convenient for artists to draw up the question scenarios because the tangram pieces remain in proportion.

(2) Game Platform

The game platform designed for this study must incorporate the following functions:

- It must be possible to mount a webcam above the platform.

- There must be an obstacle mechanism to interfere with the gameplay.

- The platform must have a tipping function to tip the actual tangram over.

To bring the platform closer to the overall style of the classroom, Fischertechnik was used to build the platform structure. This also allowed the study to take advantage of Fischertechnik's ease of assembly to easily change the overall structure and fine-tune the details during the initial design phase. The completed design is as shown in Figure 3. The webcam itself could be clipped to any object. By securing it in place with Fischertechnik avoided the player displacing the webcam so much that it cannot be used for decision-making.

Towards the latter stages of the game the obstacle mechanism comes into play and interferes with the player's attempt to assemble the tangram shape. It does not however interfere with pieces already in place. During game decision-making however the obstacle must return to its original position to avoid interference. The button function is used for the player's answer and confirm button. It is placed on the left hand side. As most players are right-handed, they can use the right hand to adjust the tangram and the left hand to answer. This helps the player save time. The tipping platform is one of the key elements in the design of this study. Apart from helping players clear the bench when they enter the next level and break up the shape, it also helps move the tangram pieces to the player's hand (Figure 4) so that no game time is wasted. Using a green 
background means that when the overall platform is shown through the webcam, it looks like a closer approximation of the blackboard. The player then feels like they are playing the game on a blackboard.

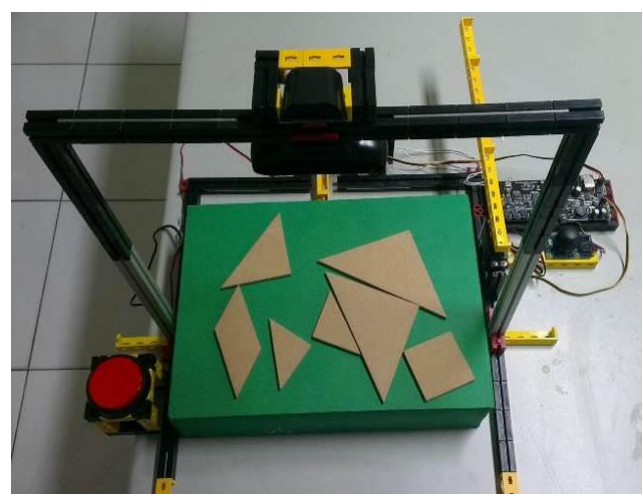

Fig. 3: Overall setup.

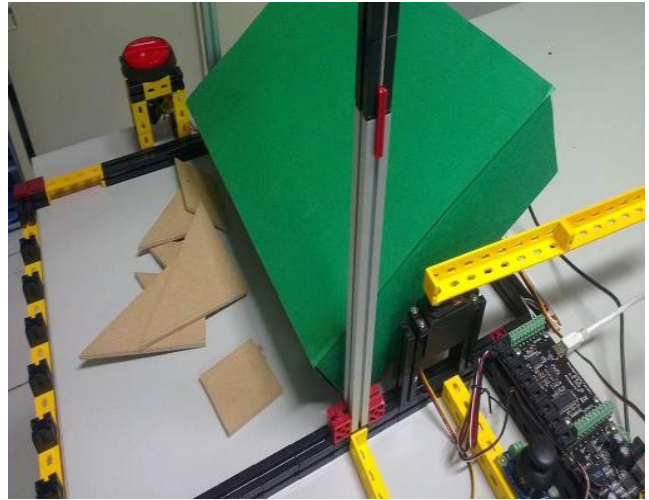

Fig. 4: Side view of the tipping platform.

While the game is in progress, the actions and display of the platform are simultaneously presented on the game screen (Figure 5). In the beginning, the player will feel a sense of disconnection between the eyes and the hand. The game obstacle does not just serve as a source of interference. Towards the end of the game when the player becomes used to the disconnect between the eyes and the hand, interference from the obstacle will force the player to pay attention to the actual platform as well, increasing the difficulty of the game towards the end.

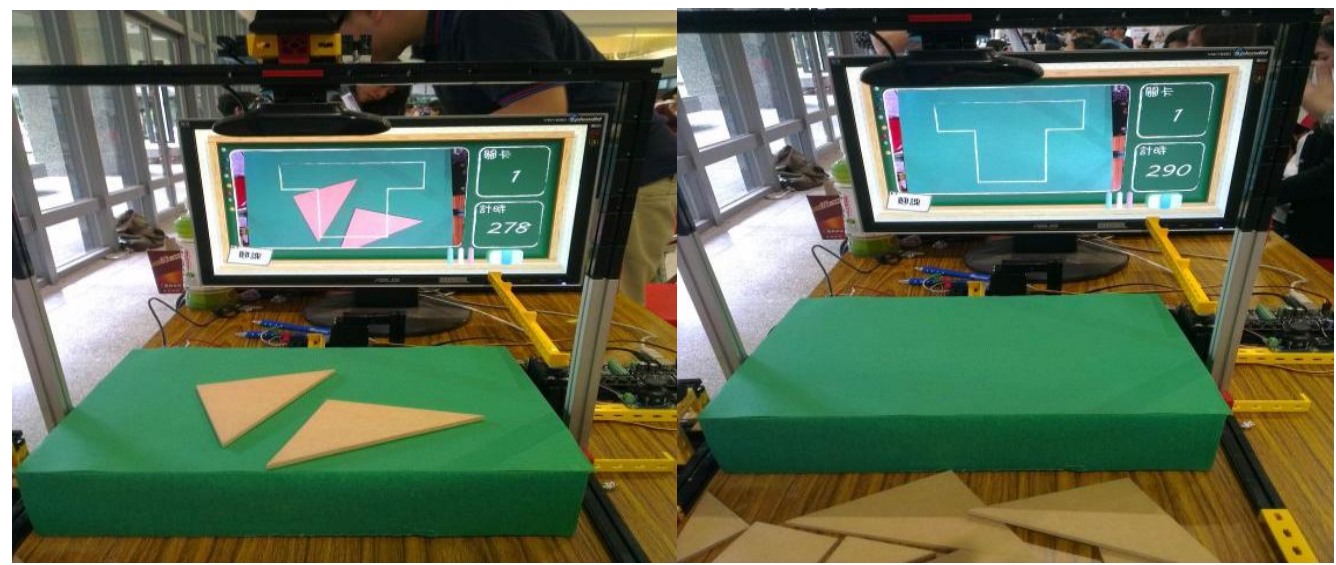

Fig. 5: Game process (illustration of overall setup).

\subsection{Game interface}

A feature of digital games is the use of art design to attract players' attention. The "After School Tangram" designed for this study uses classroom and blackboard related elements to form the overall game screen. Most of the lines are designed to have a chalk-like appearance to create a classroom ambiance for players.

The overall game interface is as shown. The top right corner shows the current level and the lower right corner shows the total timing remaining in the game. There are a total of 4 levels and the time is not reset each level. When there are 30 seconds left the count-down timer sound effect is triggered. The "Skip Class" button in the lower left corner is the answer button. It is used when the player has finished the shape or to quit the game. When pressed, the game goes into decision-making mode. Figure 7 shows the obstacles in the later levels of the game. The servo motor allows the desired angle to be easily achieved.

Game score ranking. The player score is shown on the left side of the screen while the top 5 scores are shown on the right. The scoring method is as follows:

\section{Score $=$ Time remaining + Levels completed $* 20$}


If the player failed half-way then the time is not converted into score. In other words, it's the score shown when the player completes level 1 but fails level 2. Converting time into the score makes player care about the passing of time and make them more tense about time wasted. This is what the time element is supposed to achieve.

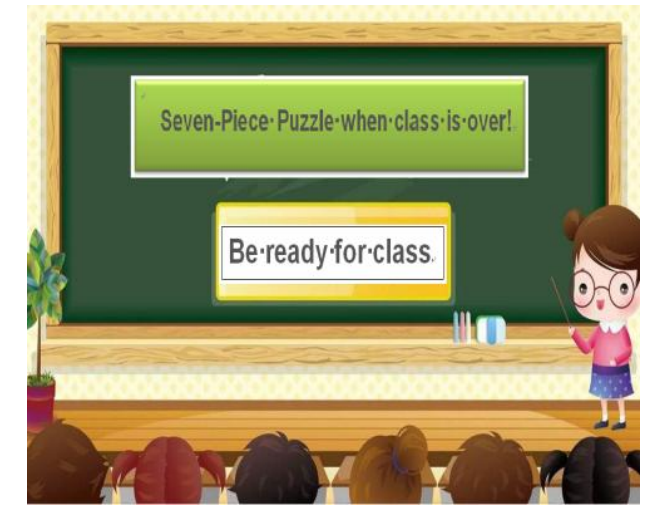

Fig. 6: Opening screen of the game.

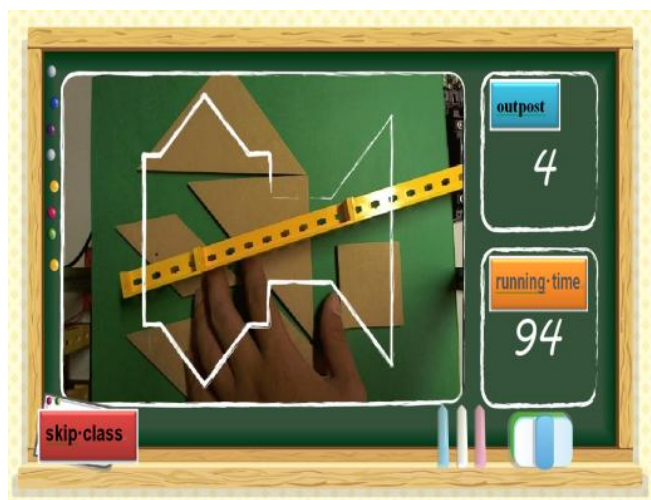

Fig. 7: Obstacle while game is in progress.

\subsection{Game evaluation and discussion}

The game designed for this study was demonstrated in a public setting after completion and questionnaires handed out. A total of 46 responses were collected with 0 invalid responses. When asked about duration of game experience, $73.9 \%$ felt it was appropriate while $19.6 \%$ felt it was too short. On game difficulty, $60.9 \%$ felt it was appropriate while $30.4 \%$ felt it was difficult. On the game's method of operation, $76.1 \%$ felt it was clear and simple. On the colors of the game pieces $54.3 \%$ felt it didn't need changing while $43.5 \%$ felt it should be increased. When players were asked to evaluate the challenges (including: different tangram shapes, complexity of tangram shape, time pressure, difficulty of level obstacle, game videoconference screen), most of the players felt that time pressure was the biggest challenge. This showed that adding time as a game element to tangram changed how its game is played. The complexity of tangram itself and the different shapes still posed a challenge to most players. On the sense of achievement that the game designed in this study offers to players (evaluation items included: completed shape, level completion screen, time remaining upon game completion, and game's method of operation), most players felt that the game gave players the greatest sense of achievement when they completed the shape. This indicated that the additional gaming or interactive elements added by this study did not impact on the inherent interest of tangram.

\section{Conclusions}

This study carried out game design based on the two directions of digital games and game interaction mechanism (game platform with game content and hardware interaction). The physical gaming console and digital game in this study used an integrated design of the software and hardware to enhance overall player operation and the fun of game play through the addition of interactive mechanism, the addition of gaming elements, and the overall presentation of digitization effects. To retain the physical aspect of the tangram, it was necessary to complement the digitization of the tangram with image recognition technology. This not only allowed the tangram to be transferred to the game screen but also the player's hand movements as well, making the game feel as if it was being played on screen. Such a complete integration of both the digital and physical playing methods not only retained the existing game mechanism but also enabled the incorporation of gaming elements that are harder to implement in the real world. The result is a digital tangram game that incorporates physical operation. The overall structure of the platform takes advantage of Fischertechnik's ease of assembly/disassembly. The playing block appearance also resembles classroom elements more closely. Mating the Phidgets sensor devices to servo motors allowed the building of a movable platform and obstacle mechanism. Adding the real-time display from the webcam increased the interactivity of the game as a whole. After much testing and refinement, adjustment of the webcam position, testing of the obstacle 
levels, and designing of the movable platform, a balance was achieved between the design philosophy and player requirements to give players an interactive puzzle games quite unlike conventional puzzle games.

\section{Acknowledgements}

The current study is supported by the Ministry of Science and Technology, Taiwan (MOST 104-2221-E$152-005-)$.

\section{References}

[1] J. Juul. The Game, the Player, the World: Looking for a Heart of Gameness. In: Copier, M., Raessens, J. (Eds.), Level up: Digital Games Research Conference Proceedings. Universiteit Utrecht. 2003, 30-45.

[2] J. Preece, Y. Rogers and H. Sharp. Interaction design: Beyond human-computer interaction. New York: John Wiley \& Sons, Inc. 2002.

[3] R. J. Pagulayan, K. Keeker, D. Wixon, R. Romero, and T. Fuller. User-centered design in games. In J. Jacko and A. Sears (Eds.), Handbook for Human-Computer Interaction in Interactive Systems. Mahwah, NJ: Lawrence Erlbaum Associates, Inc. 2003, 883-906.

[4] J. Jesper Juul. The Game, the Player, the World: Looking for a Heart of Gameness. In Level Up: Digital Games Research Conference Proceedings, edited by Marinka Copier and Joost Raessens, 30-45. Utrecht: Utrecht University, 2003.

[5] E.M. Avedon and S.S. Brian. The Study of Games. John Wiley \& Sons, Inc., New York, 1981.

[6] S.Y. Ye. Digital Gaming Design Master Class. Taipei City: Gotop Information Inc. 2010.

[7] M. Prensky. Digital Game-Based Learning. New York: McGraw-Hill, 2007.

[8] C.S. Lee et al. Human-Machine Interface Design. New Taipei City: National Open University. 1998.

[9] B.D. Rondeau. For mobile APPlications, branding is experience. Communications of the ACM, 2005, 48 (7) : 61-66.

[10] P. Booth. An introduction to human-computer interaction,” East Sussex,BN32FA, U.K.: Lawrence Erlbaum Research.1989.

[11] J. Novak. Game Development Essentials: An Introduction. Stamford," Connecticut, U.S.: Thomson Learning. 2004.

[12] V. Bellotti, M. Back, W.K. Edwards, R.E. Grinter, A. Henderson and C. Lopes. Making Sense of Sensing Systems: Five Questions for Designers and Researchers. Proceedings of the Conference on Human Factors and Computing Systems. 2002, 415-422.

[13] M.B. Holbrook. The millennial consumer in the texts of our times: Experience and Entertainment. Journal of Macromarketing, 2000, 20: 178-192.

[14] M Prensky. Digital Game-Based Learning. New York: McGraw-Hill, 2007.

[15] T. Jeng and C.H. Lee. Interaction and Social Issues in a Human-Centered-Reactive Environment. in,CAADRIA, Prentice Hall, Malaysia, 2002, pp.285-292. 